\title{
Task Scheduling Management for Load Balancing using Task Grouping Based on Cloud Computing
}

\author{
Ahmad Helmi Abdul Halim¹, Asif Iqbal Hajamydeen ${ }^{1}$ \\ ${ }^{1}$ Faculty Information Sciences and Engineering, Management and Science University \\ Shah Alam, Malaysia \\ Email: ahmadhelmiee [AT] gmail.com \\ ${ }^{1}$ Faculty Information Sciences and Engineering, Management and Science University \\ Shah Alam, Malaysia \\ Email: asif [AT] msu.edu.my
}

\begin{abstract}
Managing task scheduling management in cloud computing is an essential part for the landscape of complex procedure tasks based on various resources in a proficient and scalable path. The aim of this research is to dynamically optimize the aforesaid issue of task scheduling. The task management improvises the imperfection algorithm by pursue on weighted fair queuing model, which is significantly effective compared to the existing method. A task scheduling model has been created to demonstrate the proposed scheduler management. Study shows the improvement in the adaptation of round robin and shortest job first algorithm performing better than the existing algorithm according to the differentiate execution measurements such as, turnaround time, task size and average waiting time. In addition, context switches play an important role in algorithm by sharing between multiple tasks and running task in the scheduler. Altogether, a significant improvement between existing algorithm and proposed studies follows up accordingly to a specific context switching takes place.
\end{abstract}

Keywords-Scheduling, cloud computing, weighted-fair queue, load balancing.

\section{INTRODUCTION}

Task scheduler in cloud environment is encountering a fast advancement in term of scholarly world and it is usually stimulated by the industry point of view which decides its attention on user applications. In cloud computing environment there are several material undertaking tasks job ought to accomplish by accessible method resource which requires a capability response for the task management such as high-performance management, decrease task fulfilment time and lessened response time. Cloud computing keep the information accordingly and circulates the information in open environment based on the collected information. Task scheduling is one of exploration points of topics in cloud environment because it is consistently reliable task that challenges the assortment of task for different accessible resource in adaptable and productive way. Along these lines, task scheduling balances the cloud environment to avoid overburdening of undertaking tasks.

Many existing algorithms [2] provide balancing for load task, priority task, cost task, energy consumption to increase the accomplishment according to the performance algorithm. Load balancing priority is to improvise the load balance for all load between multiple tasks, to guarantee that every computing resource is circulated reasonably and capably. It helps in preventing the system imbalance due to the load and provides high fulfilment to the users. Provided information [4], first, discovering is to determine the number of resources that has been found in the network and to accumulate the information that are related to any similarity of scheduling management. Second, is selection, task scheduler is going through selection resources dependent on certain resources and task. Third, is task submission, where task is submitted after the collection of information has been completed and send the resources that has been chosen. This way resource selection and task determination can give outcome and provide suitable result over sequence scheduling.

A scheduler is one of the examples of decipher as a queuing framework that involve a server providing services and solution to help users in determine value. The user queue the packets for services, which then picked by the scheduler for process transmission that are reliant on the service characterized by the scheduler algorithm. A task scheduler may provide [3] as the main scheduling parameters considered in the previous method is listed to identify the scheduling attributed which are considered the most and least significant with different algorithms.

In this paper, duration is defined as the time to be completed during task scheduling that required to leave from the 
system and as the distance in time progression from the start of work to the end of the process. Deadline is characterized as the time frame for each task scheduling from submitting an undertaking task dependent on which it must be completed during that term duration. Minimum performance time is the focus for a scheduling algorithm to perform better to ensure that the system can execute the task on time. Completion time is the requirement of information need to be completed during work process from time to time for the undertaking task completion dependent on the span of time for whole execution of a job management task. This incorporates the implementation dependent on schedule and delay that are caused by cloud computing framework. Minimize the task accordingly that depend on the completion time is one of the significant things that to be considered by the current stage of platform of scheduling algorithm.

\section{RELATED WORKS}

Task scheduling is one of the considerable managements that is performed to achieve the objective of solution for the undertaking task to be monitoring and schedule accordingly to the user permissions in the computing environments. The performance of round robin scheduling [8] is subtle subject in term of time quantum elective because in comparison if time quantum that being implement is larger than round robin methodology is going to be in similar method as FCFS scheduling algorithm meanwhile, if time quantum is vice versa then round robin algorithm are going to affect the same way as algorithm and context switches is become highest. Task grouping scheduling algorithm [9] focuses on prioritization from different task is effectively reduced the processing time compared to the task scheduling that are perform without any grouping process because from perceptive based on cost of task scheduling, the task job is able to further reduce.

The suggestion of the grouping task is a result of performance that is like the scheduling management explanation because in both instances grouping task seen on parameter such as task completion time, turnaround time, and size of task to ensure the management are utilized efficiently. The average turnaround time and cost predominantly for task scheduler deceasing eventually that these two values of each task are minimized independently similarly as a total value of tasks increasing which also improved the stability of the system [4]. The combination of completion time and size of cost are affecting the task scheduling processing because if the task management are not concerned with better utilize of the scheduling that focuses on prioritization task scheduling may regulate more increasing waiting time for low priority tasks.

Priority based on dynamic resource allocate to task scheduling, which is considers as multiple service level agreement SLA objective by pre-empting best job task in cloud environment [10]. The study has been explained that hieratical scheduler makes the most of architecture for controlling effective scheduling in addition, two questions are needed to apply to ensure that the processing of the scheduling execution know on how to navigate the tasks, in what situations accordingly, and on how to schedule ahead when preparing the task management. Task job has multiplicity the priority at global situations when using load balancing for heterogeneous processors. (TDP) method [10] are one of the scheduling management to perform scheduler of the task job, " $\mathrm{T}$ " is stand as task election, " $\mathrm{D}$ " for deadline, and "P" is priority based on cost when selection task is meet its requirements, lastly, scheduling is completing by using single priority queuing. Round robin scheduling method [12] consist of time quantum to assigned multiple process, which is considered static likewise, the performance of round robin scheduling depends on heavily time quantum size and from point of view the time quantum which are smaller when using context switcher for it is more reliable comparing to the larger time quantum, which make the response time bigger.

\section{Two Ways Priority Optimization Algorithm for Task Scheduler in Cloud Environment}

Implementing the task scheduling queue structure, activities were executed on virtual operating systems and the resources are provided to the virtual system by the original system. The task is free or subordinate, and there might be few undertaking tasks whose every requirement resource is not accessible to any single data centre. The undertaking task were separated into two groups which is "accessible" and "halfway accessible". Accessible is the combination group of tasks that can be completely executing on single data centre and partially accessible are the group of undertaking tasks which is only requires for resources that requires other data centre [5].

\section{Dynamic Optimize Fair Priority Task Scheduling in Cloud Computing based of Concepts and Implementations.}

User submits the quantity of cloudlets to datacentre broker (DCB) and after that the irregular generator number determination progressively at runtime which information from task scheduling are not designation. Each value from task has various lengths interchange accordingly to the specific measure of number. Likewise, the additional length of task job management can be change progressively that depend on the execution task progression. Due date for each undertaking task is also chosen by utilizing randomly using generated numbers and therefore due date is not static. Task grouping is partitioned into two groups which is cost-based and deadline-based [2].

\section{Improvised Max-min Task Scheduling Algorithm in Cloud Computing}

Calculation algorithm corresponding to expected completion time by submission tasks from the scheduling management on each available resource. At the point where the undertaking task with high expected execution time with 
weighty task is assigned to the particular resource that has the lowest completion time and slowest on the scheduling management. Therefore, the scheduled task is being removed and every single determined time that are involve are going to overhaul and max-min algorithm method is connected to the remaining undertaking tasks for the cloud computing [6].

\section{Improvised Scheduling Task based Task Grouping for Cloud Computing}

Task schedule usually have multiple paths to send the information which contain storage server to store and computational server to running into multiple access, task group and selection resource functions are determine any acceptation requirement tasks. And group it into list and make an available selection from computing resource by dispatcher follow on information collection where the process is going to collected information for resource available from cloud information services [11].

\section{Latency aware max-min algorithm for allocate resource for cloud environment.}

The latency involvement shows broad outline that involved cloud services and cloud users. The utilization of cloud resource from consumer are not validated and usually depending according to requirement of consumer. The monitor system in this model are shows variation on utilization pattern and rely on pattern and other parameters such as availability and priorities of the resource regarding on resource assignment which made by the service provided [14].

\section{PROPOSED FRAMEWORK}

Task scheduling management is one of the fundamental focus of topic research in cloud computing consists of identified multiple job that need to be organised in one system to ensure that the flow of processing is not going to be mismanage in any additional capacity. The proposed scheduling focusing on simulation task scheduling by improving the priority of the time average and turnaround time priority for making the selection of tasks which more efficiency to existing method resources in cloud computing. Aim for this scheduler is to combine both to ensure that grouping task between time priority and cost priority based on the suitable and relevant scheduling attributes.

\subsection{Collection of data}

The round robin and shortest job first model algorithm are used to demonstrate the simulation of task scheduling and the results of the simulation are verified and taking the solution using simulation scheduling for tested. In terms of scheduling management, availability of services such as identifying the scheduling algorithm that are like the existing methods in scheduling management and an attempt to create a comparison between the algorithm also take the design of the process as an example to improvise the current design.

\subsection{Design of framework component}

The proposed scheduling focusing on simulation task scheduling by improving the priority of the average time and turnaround time priority. Aim for this scheduler is to combine both to ensure that grouping task between time priority and cost priority based on the suitable and relevant scheduling attributes.

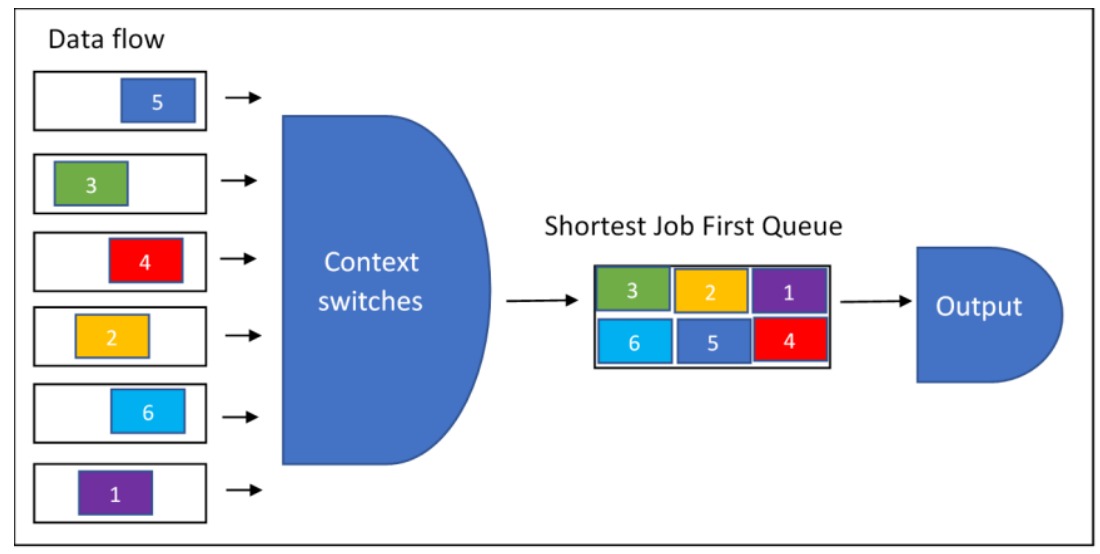

Figure 1: Process flow queuing works using Shortest Job First queuing

Number of data going through the process to determine which number are being chosen to be executed from shortest job first algorithm and by running the task schedule the number are chosen by focusing on context switches as parent to decided. 


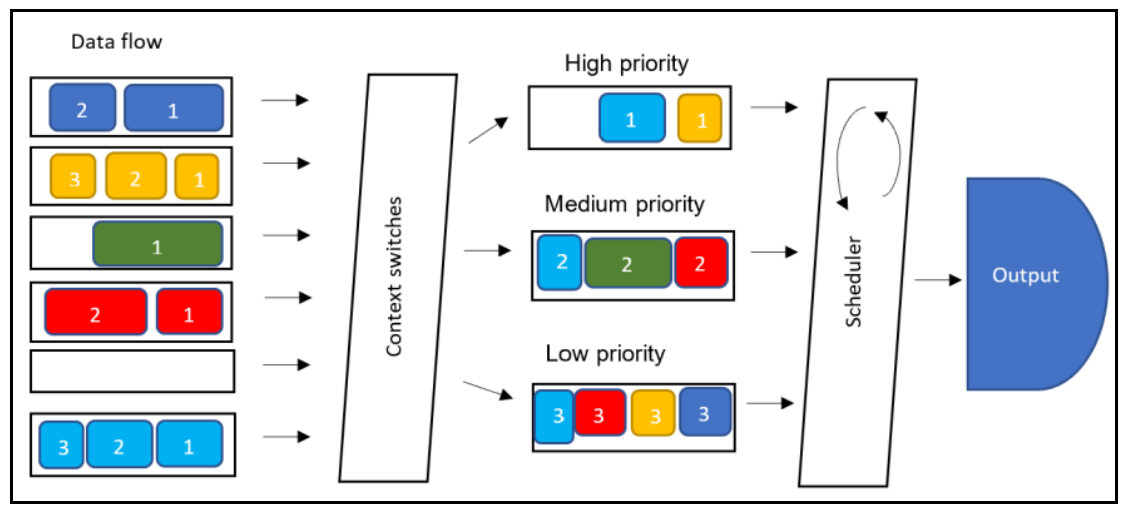

Figure 2: Weighted queue model based on combination round robin and shortest job first fashion

Combination of both algorithm into one process model, the study method applies existing improvise weighted queue model that can give stability for task scheduling by sorting into the three priority stages which is high, medium, and low priority using round-robin scheduler and shortest job first.



Figure 3: Block diagram

Block diagram shown as flow from requests tasks to final execution during task scheduling from five method flow.

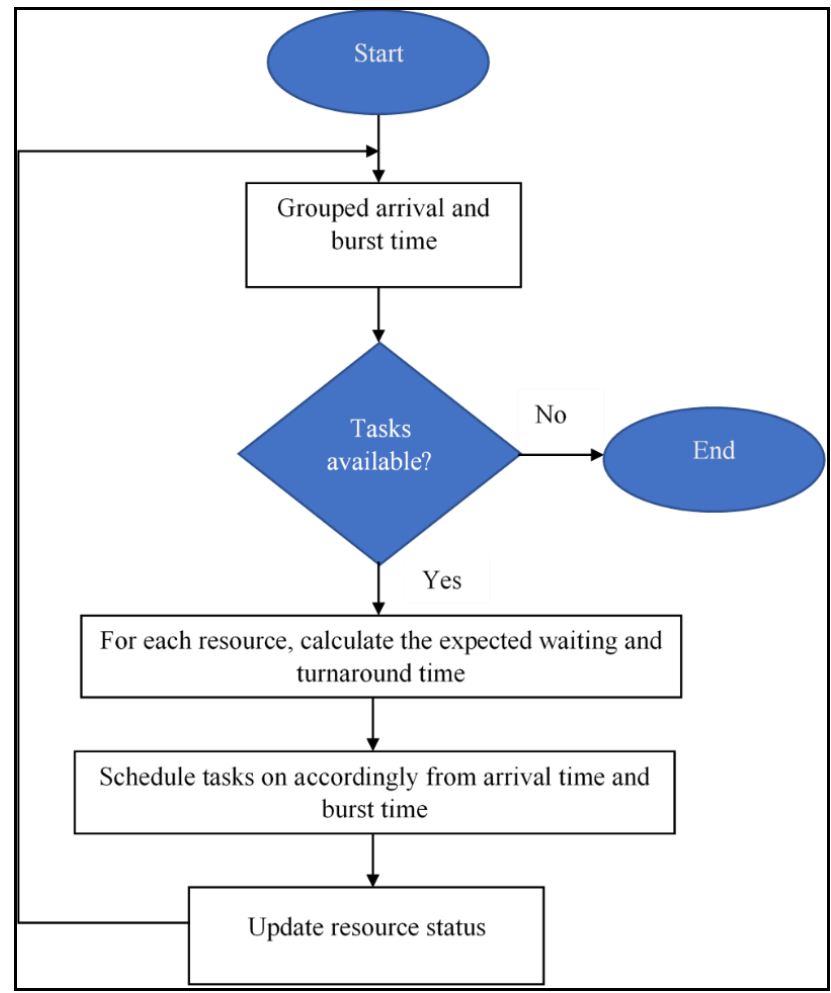

Figure 4: Flow chart of constrained task scheduling 
The flow chart shows a process of scheduling to minimize the cost of execution tasks, task scheduling algorithm aims to validate the task with minimize if it holds the capability to undertake the task. After task done execute the process, status of the process will be updated regularly.

\section{RESULT AND DISCUSSION}

The simulation results from testing for task management scheduling outlined that shows in the figures are presented and examined in detail. The output is shown using a simulation that taking advantage of previous data collecting from existing table which to consider improving the existing data to find a better algorithm method of scheduling management. The effect of task simulation testing on the performance are explained and various parameters for variable are discussed. The improvise data may found in section combination of result section which is provided with table for more understanding. Additionally, the principal goal of the simulation was to identify and make an improvement based on the objective, in what ways had to modify the original result in attempt to identify some of the improvement.

\subsection{Figures and Tables}

Table 1: Average result between algorithms

\begin{tabular}{|c|c|c|c|}
\hline \multirow{2}{*}{ Algorithm } & Waiting Time & Turnaround Time & $\begin{array}{c}\text { Number of } \\
\text { context } \\
\text { switches }\end{array}$ \\
\cline { 2 - 4 } & 33.80 & 42.80 & 11 \\
\hline Revamped Mean Round Robin & 20.8 & 29.80 & 4 \\
\hline $\begin{array}{c}\text { Bask Scht TimeQuantum Round Robin } \\
\text { Balancing Using Task Grouping Based } \\
\text { on Cloud Computing }\end{array}$ & 27.0 & 45.2 & 11 \\
\hline $\begin{array}{c}\text { Task Scheduling Management For Load } \\
\text { Balancing Using Task Grouping Based } \\
\text { on Cloud Computing }\end{array}$ & 29.0 & & 4 \\
\hline
\end{tabular}

The comparison shows that each value shown the follow up to a specific performance and going to affect three parameters which is waiting time, turnaround time and number of context switch. The various parameters are taking notes based on the selection algorithm. From the table 1, the algorithm is being taken example using the "RMRR" and "Best Time Quantum RR" [8] comparison simulation using the existing result and compare them with updated simulation with proposed studies according to waiting time, turnaround time and context switches to provide a different value.



Figure 4: Comparison value from bar chart

The bar chart implements the comparison by focusing on waiting time, turnaround time and number of context switches. Based on figure 4, it was proved to become the best and worst case of proposed algorithm for "Revamped Mean Round Robin" and "Best Time Quantum round robin" [8]. Experimental result also provided significant improvement 
between proposed studies with Revamped Mean round robin in term of average waiting time but a little disadvantage for average turnaround time. As mentioned, proposed studies show a significant improvement in term of average waiting time with "Revamped Mean Round Robin" but not to "Best Time Quantum RR".

Table 2: Comparison result between three cases

\begin{tabular}{|c|c|c|c|c|}
\hline \multirow{4}{*}{ Case 1} & Attribute & $\begin{array}{c}\text { Convention RR } \\
\text { Algorithm }\end{array}$ & $\begin{array}{c}\text { Task Scheduling Management } \\
\text { for Load Balancing Using Task } \\
\text { Grouping Based on Cloud } \\
\text { Computing }\end{array}$ & Remarks \\
\hline & Average Waiting Time & 57.2 & 38.4 & $\begin{array}{c}18.8 \text { units of time } \\
\text { saved }\end{array}$ \\
\hline & $\begin{array}{c}\text { Average Turnaround } \\
\text { Time }\end{array}$ & 76.2 & 57.8 & $\begin{array}{c}18.4 \text { unit of time } \\
\text { saved }\end{array}$ \\
\hline & Context Switch & 14 & 14 & \\
\hline \multirow{3}{*}{ Case 2} & Average Waiting Time & 140.6 & 124.8 & $\begin{array}{l}15.8 \text { units of time } \\
\text { saved }\end{array}$ \\
\hline & $\begin{array}{c}\text { Average Turnaround } \\
\text { Time }\end{array}$ & 181.2 & 169.2 & $\begin{array}{l}12 \text { unit of time } \\
\text { saved }\end{array}$ \\
\hline & Context Switch & 15 & 15 & \\
\hline \multirow{3}{*}{ Case 3} & Average Waiting Time & 65.3 & 48.83 & $\begin{array}{l}16.47 \text { units of } \\
\text { time saved }\end{array}$ \\
\hline & $\begin{array}{c}\text { Average Turnaround } \\
\text { Time }\end{array}$ & 76.66 & 67.5 & $\begin{array}{l}9.16 \text { unit of time } \\
\text { saved }\end{array}$ \\
\hline & Context Switch & 15 & 15 & \\
\hline
\end{tabular}

The comparison shows that each value shown the follow up to a specific performance and going to affect three parameters from waiting time, turnaround time and number of context switch. The various parameters are taking notes based on the selection algorithm. Based on table 2, the simulation testing has been performed with proposed studies and comparing the result studies [7] and provided with unit of time saved from the simulation experiment.

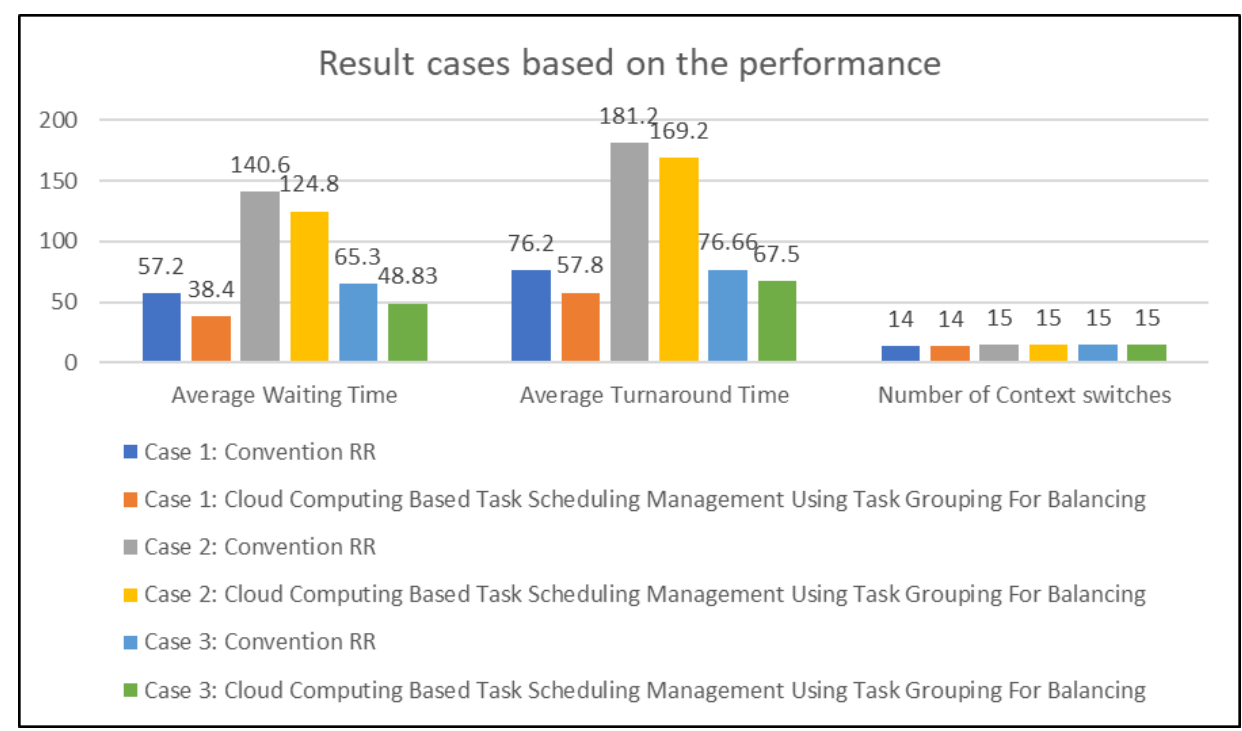

Figure 5: Result from simulation performance

Result shows according to the simulation testing which has been performed with proposed studies and comparing the result from studies which is Convention RR [7] with different cases to evaluate the result. Therefore, proposed algorithm can improve certain area such as waiting time and turnaround time using context switches to group each value to determine the improvement result. And so, according to cases provided each result shows a significant improvement between algorithm Convention RR and proposed algorithm. In conclusion, case 7, case 8 and case 9 shows proposed method able to reduce the average from waiting time and turnaround time on different cases when the values is comparing to Convention RR.

\subsection{Implementation of framework}

To perform the scheduling management, statistical method is used to demonstrate the data because method for collection is preferable suitable and appropriate to analyse, interpreting and summarize variable numerical data task 
scheduler which the information is collected and extend the information of task for scheduling can be applied to similar situations. By estimating parameters in scheduling management, one can monitoring the task job make a summarization based on calculation statistics and display the task scheduling information in form of graphs, charts or tables as well as the data can be adjusted that the results can be comparable based on the factors.

I. Grouping the remaining tasks that depend on the limitations according to the (size task, arrival-time) priority standpoint.

II. Time priority task group, to focuses on:

(i) Arrange the remaining time-based task in three queues according to their priority of high, mid and low.

(ii) Perform one given task from each priority follow up on each queue, using weighted fair queue model to utilizing the queue scheduling algorithm.

(iii) Figuring the value of minimum turnaround time using Turnaround time $=$ waiting time + (task length/processing). Likewise, calculate each tasks cost using formula of toolkit *(task length/processing). Then, the value will update the waiting time accordingly.

(iv) According to the tasks, choose toolkit with least minimum turnaround time, so that recompense time for each undertaking task can be reduced exclusively.

III. Cost priority grouping, to focuses on:

(i) Schedule each task depends on their priority from high, mid and low priority.

(ii) Execute the weighted fair queue model, and then extraction task job is going to run,

(iii) Determine the total execution time of task by execution. And, evaluate cost of each toolkit using the formula "Cost $=$ resource cost $*$ task length/processing" and then categorize them in order.

Execute task scheduling to find the minimum waiting time and turnaround time so the optimization able to execute.

\section{CONCLUSION}

Task scheduling management is a popular issue, but despite that, it seems as though no one has any real solutions to the problems. Certain critics assert that scheduling can be a little complicated and required a full point of view from certain people, while authorities in the field have said that to ensure the control of the task management. At the most basic level, it seems like each problem is making reasonable points to validate, even though the problem provides different ways to solve. Among all the available scheduling methods, the weight fair queue model to perform the task scheduling can dynamically improve the task scheduler from the bottom to top level. The study is related to the fairness balancing algorithm using weighted fair queue model than a simple priority queuing management. This fundamentally targets the information that is received from the perceptions for specific requirements, explicitly from scheduling system.

\section{ACKNOWLEDGEMENT}

The author, praise and offer gratitude to supervisor, friends and family for helping accomplish this thesis that become piece of my life. It is said that a thesis for university is resembles a voyage that can be loaded up with assortment of inclination that can occur with bliss, unpleasant and dissatisfactions. My companions who were there for me when the journey is going tough there are not enough words of extraordinary gratefulness to thank you all. I would like to symbolize my very great appreciation to all my companion and lecturer, for their unending effort in taking care throughout all my journey at Management \& Science University. They have been my inspiration and target in my study and towards the completion of this project for their happiness.

\section{REFERENCES}

[1] A. H. A. Halim and A. I. Hajamydeen, "Cloud Computing Based Task Scheduling Management Using Task Grouping for Balancing," 2019 IEEE 9th International Conference System Engineering Technology (ICSET) 2019, pp. 419-424, doi: 10.1109/ICSEngT.2019.8906508.

[2] Saxena and Chauhan. "Dynamic Optimization Fair Prioritization Task Scheduling in Cloud based Concepts and Implementations". International Journal of Computer Network and Information Security, 8(2), 2016, pp. 41-48. doi:10.5815/ijcnis.2016.02.05.

[3] Ram and Nagesh. "A Dynamic Optimization for Task Scheduler in Cloud Computing Resource Usage support". International Journal Scientific Engineering, vol. 02(10), 2013, pp. 1062-1068.

[4] Monika. "A Dynamic Optimization using Task Scheduling in Cloud Environment”. International Journal Engineering Research and Applications (IJERA), vol. 2(3), 2012, pp. 2564-2568.

[5] Shachee. "Double Level Priority based on Optimization for the Task Scheduler in Cloud Computing". International Journal Computer Application. vol. 62(20), 2013, pp. 0975-8887. 
[6] Upendra. "Improvised Max-min Task Scheduler for the Cloud Computing". International Journal Application Innovation Engineering, vol. 2(4), 2013, pp. 259-264.

[7] Sandeep. "Improved Round Robin Approach using Dynamic Time Quantum to Improving Average Waiting Time". International Journal Computer Application. vol. 69(14), 2013, pp. 12-16. doi: 10.5120/11909-8007.

[8] Khokhar "Best Time Quantum Round Robin Algorithm”. vol. 03(05), 2017, pp. 213-217.

[9] Yogita. "Dynamically optimized cost-based task scheduling for the Cloud Computing". International Journal Emerging Trends, vol. 2(3), 2013, pp. 38-42.

[10] Chandrashekhar and Rajnikant. "Priority Based Dynamic Allocation in Cloud Computing". 2012 International Symposium Cloud Services Computing. doi: 10.1109/ISCOS.2012.14.

[11] V. Vaithiyanathan. "Efficient TPD Scheduling Algorithm Cloud Environment", International Journal Engineering Technology, vol. 5(3), 2013 pp. 2030-2035. doi: 0975-4024.

[12] M. Mustafa. "Improvise Scheduling Task based Task Grouping in Cloud Environment". International Journal Computer Applications, vol. 93(8), 2014.

[13] Pallab, Biresh, Amarnath, Rahul and Ritik. "Comparative performance analysis of optimization round robin using dynamic time quantum and static time quantum based on Real Time System". International Journal Engineering Computer Science, vol. 8(12), 2019, pp. 24890-24893. doi: 0.18535/ijecs/v8i12.4399.

[14] Kashish, "latency-aware max-min algorithm for resource allocate in cloud", International Journal of Electrical and Computer Engineering (IJECE), vol. 11, no. 1, 2020 pp. 671-685. Doi: 10.11591/ijece.v11i1.pp671-685. 